Ilchmann, Achim ; Owens, David H.:

Adaptive stabilization with exponential decay

Zuerst erschienen in:

Systems \& Control Letters 14 (1990), S. 437 - 443

DOI: 10.1016/0167-6911(90)90095-C 


\section{Adaptive stabilization with exponential decay}

\section{Achim ILCHMANN}

Institut für Angewandte Mathematik, Universität Hamburg, D2000 Hamburg 13, West Germany

\section{David H. OWENS}

Dynamics and Control Division, Weir Building, University of Strathclyde, Glasgow GI $1 X J$, Scotland

Received 4 November 1989

Revised 29 January 1990

Abstract: Adaptive high gain stabilizers for classes of linear time-invariant state space systems are presented. The classes cover multi-input-multi-output systems where the state dimension is not known. Only standard assumptions such as minimum phase and known respectively unknown sign are required. The main result is, that the adaptive control laws of Byrnes and willems can be modified to produce a guarantee of exponentially decaying states.

Keywords: Adaptive stabilization; adaptive control; linear time-invariant systems; robustness; global adaptive controllers.

\section{Nomenclature}

$$
\begin{aligned}
& \mathbb{R}_{+}=\{s \in \mathbb{R} \mid s>0\} . \\
& \mathbb{C}_{-}=\{s \in \mathbb{C} \mid \operatorname{Re} s>0\} . \\
& \mathbb{C}_{-}=\{s \in \mathbb{C} \mid \operatorname{Re} s<0\} . \\
& \sigma(A)=\left\{\lambda_{1}(A), \ldots, \lambda_{n}(A)\right\}=\text { spectrum of } A \in \\
& \mathbb{R}^{n \times n} . \\
& L_{r}(0, t)=\text { set of all r-integrable functions } f:
\end{aligned}
$$
$\mathbb{R}^{n \times n}$ $(0, t) \rightarrow \mathbb{R}^{n}$

$L_{\infty}(0, t)=$ set of all bounded functions $f$ : $(0, t) \rightarrow \mathbb{R}^{n}, n$ being defined by the context.

$\mathscr{C}\left([-h, 0], \mathbb{R}^{n}\right)=$ set of all continuous functions $f:[-h, 0\} \rightarrow \mathbb{R}^{n}$.

\section{Introduction}

The problems of adaptively stabilizing a linear system by dynamic output feedback ( $n$ ot based on parameter identification algorithms) have received a good deal of attention in recent years. However, almost all discussed stabilizers produce a stable output $y(\cdot)$ in the weak sense, that $y(t)$ goes asymptotically to zero. The purpose of the present paper is to show, that without restricting the class of systems under consideration, it is possible to produce stronger results, namely exponential decay of the output $y(t)$, and the system state $x(t)$.

We consider the class of time-invariant linear systems of the form

$$
\begin{aligned}
& \dot{x}(t)=A x(t)+B u(t), \\
& y(t)=C x(t),
\end{aligned}
$$

where $A \in \mathbb{C}^{n \times n}, B, C^{\mathrm{T}} \in \mathbb{C}^{n \times m}$, are unknown, but have to satisfy the high frequency gain condition

det $C B \neq 0$

and the minimum phase condition

$\operatorname{det}\left[\begin{array}{cc}s I-A & -B \\ C & 0\end{array}\right] \neq 0 \quad$ for all $s \in \overline{\mathbf{C}}_{+}$.

The state dimension $n$ of the system need not be known.

For multi-input-multi-output systems, we will consider the known sign case when (1.2) is strengthened to

$\sigma(C B) \subset \overline{\mathbb{C}}_{+}$.

(Note: the case of $\sigma(C B) \subset \overline{\mathbb{C}}_{-}$is a trivial extension of this case obtained by input scaling.) For single-input-single-output systems we also consider the unknown sign case $c b \neq 0$.

Our approach is a modification of the so called Byrnes-Willems controller which, in the case of (1.4), is just

$$
\begin{aligned}
& \dot{k}(t)=\|y(t)\|^{2}, \quad k(0)=k_{0} \in \mathbb{R}, \\
& u(t)=-k(t) y(t) .
\end{aligned}
$$

Byrnes and Willems [1] have shown that the control strategy (1.5) produces an asymptotically stable output $y(t)$ if it is applied to an arbitrary system $(A, B, C)$ belonging to the class (1.1)-(1.3) with spectrum of $C B$ in $\mathbf{C}_{+}$. In this situation the sign of $C B$ is crucial and hence, if it is not known, the control scheme must find this sign. For the 
class of single-input-single-output systems which satisfy the minimum phase condition (1.3) and the unknown sign condition (1.2), Willems and Byrnes [1] presented a modification of (1.5) which also produces an asymptotically decaying output $y(t)$. They used a switching function $S: \mathbb{R} \rightarrow \mathbb{R}$, originated by Nussbaum [9], to modify the control scheme as follows:

$\dot{k}(t)=y(t)^{2}, \quad k(0)=k_{0} \in \mathbb{R}$,

$u(t)=-S(k(t)) k(t) y(t)$.

The purpose of the switching function is to search the appropriate sign for the feedback. Mårtensson [7] extended this to multi-input-multi-output systems. Robustness properties of the control law (1.5) and (1.6) are proved in [12] and [11], respectively.

If a linear system (1.1) satisfies the minimum phase property (1.3), then obviously there exists some $\omega>0$ such that

$\operatorname{det}\left[\begin{array}{cc}s I-A & B \\ C & 0\end{array}\right] \neq 0$

for all $s \in\{s \in \mathbb{C} \mid \operatorname{Re} s<-\omega\}$.

Logemann [6] used this information and showed that in the case of $c b \neq 0$, the adaptive scheme

$\dot{k}(t)=\left|\mathrm{e}^{\omega t} y(t)\right|, \quad k(0)=k_{0} \in \mathbb{R}$,

$u(t)=-S(k(t)) k(t) y(t)$,

produces an exponentially decaying output $y(t)$. Within Logeman's framework, it can be shown that in the case of $c b>0$, the adaptive scheme

$\dot{k}(t)=\left|\mathrm{e}^{\omega t} y(t)\right|^{2}, \quad k(0)=k_{0} \in \mathbb{R}$,

$u(t)=-k(t) y(t)$.

produces the highly desirable property that the output $y(t)$ is exponentially decaying. However, within his framework, he can only consider systems which satisfy the strengthened minimum phase condition (1.7) for a fixed $\omega>0$. Our strategy is different in that we propose a timevarying $\omega(t)$ in (1.8) which adaptively converges to

$\lim _{t \rightarrow \infty} \omega(t)=\omega_{\infty}>0$,

and as a consequence ensure exponential stabilization. That this is in fact possible, in both the known and the unknown sign cases, is the main contribution of the present paper.

It is noteworthy, that Miller and Davidson [8] have presented a completely different adaptive control strategy to provide Lyapunov stability for a class of systems which cover our class.

In Section 2, we present an adaptive control scheme which produces exponential decaying outputs for multi-input-multi-output systems which are minimum phase and where the 'sign' of the high frequency gain $C B$ is known. In Section 3, the analogous problem is solved for single-input single-output, minimum phase systems where the sign of $c b$ is unknown.

\section{The multi-input multi-output, known sign case}

In this section, we consider the class $\Sigma$ of systems of the form (1.1) which satisfy (1.3) with (1.2) strengthened by the requirement that the spectrum of $C B$ lies in $\mathbb{C}_{+}$.

The following lemma gives a deeper insight into these assumptions and is of technical use as well.

Lemma 2.1. Suppose $(A, B, C)$ satisfy the conditions (1.1)-(1.3) and the linear output feedback

$u(t)=-k(t) y(t)$.

where $k(\cdot): \mathbb{R}_{+} \rightarrow \mathbb{R}$ is a piecewise continuous function, is applied to the system. Then the closed-loop system

$\dot{x}(t)=[A-k(t) B C] x(t)$

can be converted, by a suitable coordinate transformation, into the form

$\dot{y}(t)=\left[A_{1}-k(t) C B\right] y(t)+A_{2} \eta(t)$,

$\dot{\eta}(t)=A_{3} y(t)+A_{4} \eta(t)$,

where $A_{1} \in \mathbb{R}^{m \times m}, \quad A_{2}, \quad A_{3}^{\mathrm{T}} \in \mathbb{R}^{m \times(n-m)}, \quad A_{4} \in$ $\mathbb{R}^{(n-m) \times(n-m)}$ and

$\sigma\left(A_{4}\right) \subset \mathbb{C}_{-}$.

Moreover, if $k(t)$ is monotonically increasing to infinity as $t \rightarrow t^{\prime}, t^{\prime} \leq \infty$, and the spectrum of $C B$ lies in $\mathbb{C}_{+}$, then

$$
\begin{aligned}
\|\phi(t, s)\| \leq & M \mathrm{e}^{\left(-\varepsilon+\delta_{0}\right)(t-s)} \\
& \text { for all } t^{\prime}>t \geq s \geq t_{0},
\end{aligned}
$$


where $\phi(\cdot, \cdot)$ denotes the transition matrix of $(2.1)$, $M>0$ is some constant independent of $t_{0}, \varepsilon>0$ so that $\operatorname{Re} \lambda_{i}\left(A_{4}\right)<-\varepsilon$ from all $i \in\{1,2, \ldots, n\}$ and $\delta_{0}=\delta\left(t_{0}\right)>0$ is monotonically decreasing to zero as $k\left(t_{0}\right)$ tends to infinity.

Proof. In Owens, Chotai and Abiri [10] it is shown that

$Q:=\left[B(C B)^{-1}, V\right] \in G L_{n}(\mathbb{R})$,

with $V$ a basis matrix of ker $C$ yields

$C Q=\left[\begin{array}{ll}I_{m} & 0\end{array}\right], \quad Q^{-1} B=\left[\begin{array}{c}C B \\ 0\end{array}\right]$

$Q^{-1} A Q=\left[\begin{array}{ll}A_{1} & A_{2} \\ A_{3} & A_{4}\end{array}\right]$

where $A_{1}, A_{2}, A_{3}$ and $A_{4}$ are of dimension $m \times m$, $m \times(n-m), \quad(n-m) \times m$ and $(n-m) \times(n-$ $m)$, resp. Now (2.2) is obvious. (2.3) follows from (1.3) and the proof of Theorem 2.2 in Ilchmann, Owens and Prätzel-Wolters [5]. (2.4) follows from (2.3) and $\lim _{t \rightarrow t^{\prime}} k(t)=\infty$; it can be deduced from the proof of the Interconnections Lemma in [4], p. $107 / 8$.

Now we are in a position to state the main result of this section on exponential stabilization.

Proposition 2.2. Suppose $(A, B, C) \in \Sigma$ and $h \geq 0$, $x_{0} \in \mathbb{R}^{n}$. Then the control scheme

$$
\begin{array}{ll}
\dot{k}(t)=\mathrm{e}^{2 \omega(t) t}\|y(t)\|^{2}, & k(0)=k_{0} \geq 0, \\
\omega(t)= \begin{cases}1 & \text { for } t \in[0, h), \\
\frac{1}{1+k(t-h)} & \text { for } t \geq h .\end{cases} \\
u(t)=-k(t) y(t),
\end{array}
$$

produces an exponentially decaying solution of the closed loop system

$$
\begin{aligned}
& \dot{x}(t)=[A-k(t) B C] x(t), \quad x(0)=x_{0}, \\
& y(t)=C x(t),
\end{aligned}
$$

and the gain converges to a finite limit $k_{\infty}$, i.e.

$\lim _{t \rightarrow \infty} k(t)=k_{\infty}<\infty$

Note that (2.6) is a nonlinear differential equation which is of delay type if $h>0$. We include it as a possibility since it makes computations easier and provides as indication of the robustness of the result to the adaptation rule. We allow the possibility that $h=0$.

Proof. We only give a proof for $h>0$; the case $h=0$ is shown in a similar manner. Using the notation

$x_{t}(\cdot):=x(t+\cdot), \quad \omega_{t}(\cdot):=\omega(t+\cdot)$,

(2.6) reads

$\dot{x}(t)=f\left(t, x_{2}\right), \quad x(0)=x_{0}$,

where

$f\left(t, x_{t}\right)=$
$\left\{\begin{array}{l}A x_{t}(0) \quad \text { for } t<0, \\ {\left[A-\int_{-t}^{0}\left\|\mathrm{e}^{t+s} C x_{t}(s)\right\|^{2} \mathrm{~d} s \cdot B C\right] x_{t}(0)} \\ \text { for } t \in[0, h), \\ {\left[A-\int_{-t}^{0}\left\|\mathrm{e}^{\omega_{t}(s)(t+s)} C x_{t}(s)\right\|^{2} \mathrm{~d} s \cdot B C\right] x_{t}(0)} \\ \text { for } t \geq h ;\end{array}\right.$

$f\left(\cdot, x_{t}\right)$ may be discontinuous at $t=h$. However, without restriction of generality we may assume that $k_{0}=0$. This can be done since $f\left(\cdot, x_{t}\right)$ satisfies the Caratheodory condition (cf. Hale [2], p. $55)$ and existence and uniqueness of (2.8) goes through as for the case of continuous $f\left(\cdot, x_{7}\right)$.

We proceed in several steps and prove the following statements:

(i) If there exists a solution of (2.8) on $\left(-\infty, t^{\prime}\right), t^{\prime} \leq \infty$, then $k(\cdot) \in L_{\infty}\left(0, t^{\prime}\right)$.

(ii) (2.8) does not have a finite escape time.

(iii) A solution of (2.8) exists on the whole of $\mathbb{R}$ and is unique.

(iv) There exist $m, \omega>0$ such that the solution $x(\cdot)$ of $(2.6)$ satisfies

$\|x(t)\| \leq M \mathrm{e}^{-\omega t} \quad$ for all $t \geq 0$

and (2.7) is fulfilled.

(i): Suppose there exists a solution $x(\cdot)$ of (2.8) on $\left(-\infty, t^{\prime}\right)$ and $k(\cdot) \notin L_{\infty}\left(0, t^{\prime}\right)$. Then Lemma 2.1 implies that

$\|x(t)\| \leq K \mathrm{e}^{-\lambda t}$ for all $t \in\left[0, t^{\prime}\right)$ 
for some $K, \lambda>0$. Since $\lim _{t \rightarrow t^{\prime}} \omega(t)=0,(2.10)$ applied to

$k(t)=\int_{0}^{t}\left\|\mathrm{e}^{\omega(s) s} C x(s)\right\|^{2} \mathrm{~d} s+k_{0}$

yields that $k(\cdot) \in L_{\infty}\left(0, t^{\prime}\right)$. Hence (i) is proved.

(ii): Suppose there exists some $t^{\prime}>0$ such that

$\limsup _{t \rightarrow t^{\prime}}\|x(t)\|=\infty$.

Since by (i), $k(\cdot) \in L_{\infty}\left(0, t^{\prime}\right),(2.6)$ satisfies a global Lipschitz condition on $\left(0, t^{\prime}\right)$. The contradicts (2.11), whence (ii) is proved.

(iii): Consider the continuous map

$f: \Omega=(-\infty, h) \times \mathscr{C}\left([-h, 0], \mathbb{R}^{n}\right) \rightarrow \mathbb{R}^{n}$

given by (2.9). $f$ is called locally Lipschitz in the second argument if $f$ is Lipschitz in the second argument in each compact set in $\Omega$. $f$ is in fact locally Lipschitz as a sum, product and composition of locally Lipschitz maps. Therefore, Hale [2], p. 42 can be applied and existence and uniqueness of the solution $x(t)$ of (2.8) through the initial value $\left(0,\left.x(\cdot)\right|_{[-h, 0]}\right)$ follows for $t \in(-\infty, \varepsilon)$, for some $\varepsilon>0$. Now (ii) implies that the solution can be continued on $(-\infty, h]$. The same arguments yield existence and uniqueness on $(-\infty, 2 h]$. By repeating these arguments inductively, (iii) follows.

(iv): Put $t^{\prime}=\infty$ in the proof of (i). Then (i) shows that $k(\cdot)$ is bounded which proves $(2.7)$. Furthermore,

$\lim _{t \rightarrow \infty} \omega(t)=\omega_{\infty}>0$.

Therefore $\mathrm{e}^{\omega(\cdot) \cdot} y(\cdot) \in L_{2}(0, \infty)$ and $\hat{y}(\cdot):=\mathrm{e}^{\bar{\omega}} y(\cdot) \in L_{2}(0, \infty)$ for all $\bar{\omega} \leq \omega_{\infty}$.

Suppose that (2.6) is written in the form (2.2). Then a straightforward calculation yields

$\frac{\mathrm{d}}{\mathrm{d} t} \hat{\eta}(t)=\left[\bar{\omega} I_{n-m}+A_{4}\right] \hat{\eta}(t)+A_{3} \hat{y}(t)$

where

$\hat{\eta}(t):=\mathrm{e}^{\bar{\omega} t} \eta(t)$.

Since $\sigma\left(A_{4}\right) \subset \mathbb{C}_{-},(2.12)$ applied to (2.13) gives $\hat{\eta}(\cdot) \in L_{2}$ for

$\bar{\omega}+\max _{1 \leq i \leq n} \operatorname{Re} \lambda_{i}\left(A_{4}\right)<0$.
Now it follows from

$$
\begin{aligned}
\frac{\mathrm{d}}{\mathrm{d} t}\left[\begin{array}{l}
\hat{y}(t) \\
\hat{\eta}(t)
\end{array}\right]= & {\left[\omega I_{n}+\left[\begin{array}{cc}
A_{1}-k(t) c b & A_{2} \\
A_{3} & A_{4}
\end{array}\right]\right] } \\
& \cdot\left[\begin{array}{l}
\hat{y}(t) \\
\hat{\eta}(t)
\end{array}\right]
\end{aligned}
$$

that $(\mathrm{d} / \mathrm{d} t) \hat{y}(t) \in L_{2}(0, \infty)$ resp. $(\mathrm{d} / \mathrm{d} t) \hat{\eta}(t) \in$ $L_{2}(0, \infty)$. Thus it follows (see, for instance, Helmke and Prätzel-Wolters [3]) that $\lim _{t \rightarrow \infty} \hat{y}(t)=0$, resp. $\lim _{t \rightarrow \infty} \hat{\eta}(t)=0$. This proves boundedness of $\hat{y}(\cdot)$ resp. $\hat{\eta}(\cdot)$ and hence proves exponential decay of $y(t)$ resp. $\eta(t)$. Therefore, the proof of (iv) is complete.

\section{The single-input-single-output, $n$-th order, un- known sign case}

In this section we consider the class $\hat{\Sigma}$ of single-input-single-output systems of the form

$\dot{x}(t)=A x(t)+b u(t)$,

$y(t)=c x(t)$,

where $A \in \mathbb{R}^{n \times n}, b, c^{\mathrm{T}} \in \mathbb{R}^{n}$ are unknown, but are known to satisfy

$c b \neq 0$,

$\operatorname{det}\left[\begin{array}{cc}s I_{n}-A & b \\ c & 0\end{array}\right] \neq 0 \quad$ for all $s \in \overline{\mathbb{C}}_{+}$.

Since the sign of the feedback gain $c b$ is not known, we have to introduce a switching function, as in Nussbaum [9] and Willems and Byrnes [13].

Definition 3.1. A piecewise continuous function $S: \mathbb{R} \rightarrow \mathbb{R}$ is called a switching function, if for some $a \in \mathbb{R}$ it satisfies

$\sup _{x>a} \frac{1}{x-a} \int_{a}^{x} S(\tau) \tau \mathrm{d} \tau=+\infty$

and

$\inf _{x>a} \frac{1}{x-a} \int_{a}^{x} S(\tau) \tau \mathrm{d} \tau=-\infty$.

The function $N(t):=S(t) t$ is called the Nussbaum gain.

Remark 3.2. (i) It is easily seen that if (3.4) resp. (3.5) holds true for some $a \in \mathbb{R}$ then it is valid for all $a \in \mathbb{R}$. 
(ii) It is also easily shown that (3.4) and (3.5) are equivalent to

$\sup _{x>a}\left[a x+\beta \int_{a}^{x} S(\tau) \tau \mathrm{d} \tau\right]=+\infty$

and

$\sup _{x>a}\left[a x+\beta \int_{a}^{x} S(\tau) \tau \mathrm{d} \tau\right]=-\infty$

for all $\alpha, \beta \in \mathbb{R}, \beta \neq 0$.

(iii) Switching functions are for instance

$$
S(\tau)=\sin \sqrt{|\tau|}
$$

or

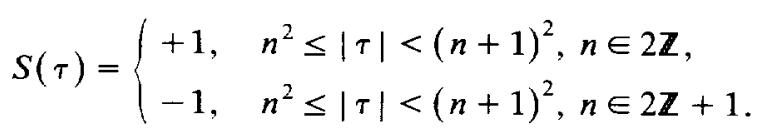

The switching function is implemented into the feedback loop using the control law

$u(t)=-N(k(t)) y(t)$.

The main result of this section is:

Proposition 3.3. Suppose $(A, b, c) \in \tilde{\Sigma}, h \geq 0$, $x_{0} \in \mathbb{R}^{n}$, and $S: \mathbb{R} \rightarrow \mathbb{R}$ is a switching function. Then the control scheme

$\dot{k}(t)=\left|\mathrm{e}^{\omega(t) t} y(t)\right|^{2}, \quad k(0)=k_{0} \geq 0$,

$\omega(t)= \begin{cases}1 & \text { for } t \in[0, h], \\ \frac{1}{1+k(t-h)} & \text { for } t \geq h\end{cases}$

$u(t)=-S(k(t)) k(t) y(t)$,

produces an exponentially decaying solution $x(\cdot)$ of the closed system equation

$\dot{x}(t)=[A-S(k(t)) k(t) b c] x(t), \quad x(0)=x_{0}$,

and the gain converges to a finite limit $k_{\infty}$, i.e.

$\lim _{t \rightarrow \infty} k(t)=k_{\infty}<\infty$

Proof. We proceed in a similar manner to the proof of Proposition 2.2 and show the following statements:

(i) If there exists a solution of (3.7) on $\left(-\infty, t^{\prime}\right), t^{\prime} \leq \infty$, then $k(\cdot) \in L_{\infty}\left(0, t^{\prime}\right)$. (ii) (3.7) does not have a finite escape time.

(iii) A solution of (3.7) exists on the whole of $\mathbb{R}$ and is unique.

(iv) There exists $M, \omega>0$ such that the solution $x(\cdot)$ of $(3.7)$ satisfies

$\|x(t)\| \leq M \mathrm{e}^{-\omega t} \quad$ for all $t \geq 0$

and (3.8) is fulfilled.

(i): Suppose there exists a solution $x(\cdot)$ of (3.7) on $\left(-\infty, t^{\prime}\right)$ and $k(\cdot) \notin L_{\infty}\left(0, t^{\prime}\right)$. Because of Lemma 2.1 we may assume that (3.7) is of the form

$\dot{y}(t)=\left[A_{1}-S(k(t)) k(t) c b\right] y(t)+A_{2} \eta(t)$,

$\dot{\eta}(t)=A_{3} y(t)+A_{4} \eta(t)$,

with $\sigma\left(A_{4}\right) \subset \mathbb{C}_{-}$. Using this, it follows that

$$
\begin{aligned}
& \frac{1}{2} \frac{\mathrm{d}}{\mathrm{d} t}\left(\mathrm{e}^{2 \omega(t) t} y(t)^{2}\right) \\
& =\dot{k}(t)\left[t \frac{\mathrm{d}}{\mathrm{d} t} \omega(t)+\omega(t)\right. \\
& \left.\quad+A_{1}-S(k(t)) k(t) c b\right] \\
& \quad+\mathrm{e}^{2 \omega(t) t} y(t) A_{2} \eta(t) .
\end{aligned}
$$

We use the notation

$\hat{y}(t)=\mathrm{e}^{\omega(t) t} y(t), \quad \hat{\eta}(t)=\mathrm{e}^{\omega(t) t} \eta(t)$.

Integration of (3.10) yields

$$
\begin{aligned}
& \frac{\hat{y}(t)^{2}-\hat{y}(0)^{2}}{2} \\
& =\int_{0}^{t} \dot{k}(\tau)\left[\left.\tau \frac{\mathrm{d}}{\mathrm{d} s} \omega(s)\right|_{s=\tau}+\omega(\tau)+A_{1}\right] \mathrm{d} \tau \\
& \quad-c b \int_{0}^{t} S(k(\tau)) k(\tau) \dot{k}(\tau) \mathrm{d} \tau \\
& \quad+\int_{0}^{t} \mathrm{e}^{\omega(s) s} y(s) A_{2} \mathrm{e}^{\omega(s) s} \eta(s) \mathrm{d} s .
\end{aligned}
$$

Since $(\mathrm{d} / \mathrm{d} t) \omega(t) \leq 0, \dot{k}(t) \geq 0$, and $\lim _{t \rightarrow t^{\prime}} \omega(t)$ $=0$, there exists some $M_{1}>0$ such that

$$
\begin{aligned}
\frac{\hat{y}(t)^{2}-\hat{y}(0)^{2}}{2} \leq & M_{1}\left[k(t)-k_{0}\right] \\
& -c b \int_{k_{0}}^{k(t)} S(\mu) \mu \mathrm{d} \mu \\
& +M_{1} \int_{0}^{t}|\hat{y}(s)| \cdot\|\hat{\eta}(s)\| \mathrm{d} s,
\end{aligned}
$$


where we have changed variables in the second summand of (3.11).

Since $\sigma\left(A_{4}\right) \subset C_{-}$, there exists some $M_{2}, \varepsilon>0$ such that

$\left\|\mathrm{e}^{A_{4} t}\right\| \leq M_{2} \mathrm{e}^{-\varepsilon t} \quad$ for all $t \geq 0$.

From (3.9) it follows that for $\eta(0)=\eta_{0}$ we have

$$
\begin{aligned}
\mathrm{e}^{\omega(t) t} \eta(t)= & \mathrm{e}^{\left[A_{4}+\omega(t) I_{n-1}\right] t} \eta_{0} \\
& +\int_{0}^{t} \mathrm{e}^{\left[A_{4}+\omega(t) I_{n-1}(t-s)\right.} \mathrm{e}^{\omega(t) s} y(s) \mathrm{d} s .
\end{aligned}
$$

Since $\omega(t)$ is monotonically decreasing to zero, (3.13) applied to (3.14) yields for some $M_{3}, \bar{\varepsilon}>0$,

$$
\|\hat{\eta}(t)\| \leq M_{3} \mathrm{e}^{-\bar{\varepsilon} t}+M_{3} \int_{0}^{t} \mathrm{e}^{-\bar{\varepsilon}(t-s)}|\hat{y}(s)| \mathrm{d} s .
$$

Since the operator

$$
\begin{aligned}
L: L_{2}(0, \infty) & \rightarrow L_{2}(0, \infty), \\
u(\cdot) & \mapsto\left(t \mapsto \int_{0}^{t} \mathrm{e}^{-\bar{\varepsilon}(t-s)} u(s) \mathrm{d} s\right)
\end{aligned}
$$

is bounded, an application of the CauchySchwartz inequality gives for some $M_{4}, M_{5}>0$,

$$
\begin{aligned}
\int_{0}^{t}|\hat{y}(s)| \cdot\|\hat{\eta}(s)\| \mathrm{d} s \\
\leq M_{3} \int_{0}^{t}|\hat{y}(s)| \mathrm{e}^{-\bar{\varepsilon} s} d s \\
\quad+M_{3} \int_{0}^{t}|\hat{y}(s)| \cdot L(|\hat{y}(\cdot)|)(s) \mathrm{d} s \\
\leq 2 M_{3} M_{4}\left[\int_{0}^{t} \hat{y}(s)^{2} \mathrm{~d} s\right]^{1 / 2} \\
\quad+M_{3}\|L\| \int_{0}^{t} \hat{y}(s)^{2} \mathrm{~d} s \\
\leq\left(M_{3} M_{4}\right)^{2}+\int_{0}^{t} \hat{y}(s)^{2} \mathrm{~d} s \\
\quad+M_{3}\|L\| \int_{0}^{t} \hat{y}(s)^{2} \mathrm{~d} s \\
\leq M_{5}+M_{5} \int_{0}^{t} \hat{y}(s)^{2} \mathrm{~d} s .
\end{aligned}
$$

Finally, by inserting (3.16) into (3.12), we obtain

$$
\begin{aligned}
& \frac{\hat{y}(t)^{2}-\hat{y}(0)^{2}}{2} \\
& \quad \leq M_{1} M_{5}+M_{1}\left(1+M_{5}\right)[k(t)-k(0)] \\
& \quad-c b \int_{k(0)}^{k(t)} S(\mu) \mu \mathrm{d} \mu,
\end{aligned}
$$

which is equivalent to

$$
\begin{aligned}
0 \leq \hat{y}(t)^{2} \leq & 2 M_{1} M_{5}+\hat{y}(0)^{2} \\
& +2 M_{1}\left(1+M_{5}\right)[k(t)-k(0)] \\
& -2 c b \int_{k(0)}^{k(t)} S(\mu) \mu \mathrm{d} \mu .
\end{aligned}
$$

Since $k(t)$ tends to $\infty$ as $t \rightarrow t^{\prime}$, Remark 3.2(ii) applied to (3.17) yields a contradiction. Therefore, (i) is proved.

The proofs of (ii)-(iv) are analogous to those of Proposition 2.2. The details are omitted for brevity.

\section{References}

[1] C.I. Byrnes and J.C. Willems, Adaptive stabilization of multivariable linear systems, Proc. of the 23rd Conf. on Decision and Control, Las Vegas, NV (1984) 1574-1577.

[2] J. Hale, Theory of Functional Differential Equations, (Springer-Verlag, New York, 1977).

[3] U. Helmke and D. Prätzel-Wolters, Stability and robustness properties of universal adaptive controllers for first order linear systems, Internat. J. Control 48 (1988) 11531182.

[4] A. Ilchmann, Contributions to Time-Varying Linear Control Systems, Thesis (Verlag an der Lottbek, Hamburg, 1989).

[5] A. Ilchmann, D.H. Owens and D. Prätzel-Wolters, High gain robust adaptive controllers for multivariable systems, Systems Control Lett. 8 (1987) 397-404.

[6] H. Logemann, Adaptive exponential stabilization for a class of nonlinear retarded processes, Math. Control Signals Systems (1990) to appear.

[7] B. Mårtensson, Adaptive Stabilization, Doctoral Dissertation, Lund Institute of Technology (1986).

[8] D.E. Miller and E.J. Davidson, An adaptive controller which provides Lyapunov stability, IEEE Trans. Autom. Conirol 34 (6) (1989) 599-609.

[9] R.D. Nussbaum, Some remarks on a conjecture in parameter adaptive control, Systems Control Lett. 3 (1983) 243246.

[10] D.H. Owens, A. Chotai and A. Abiri, Parametrization and approximation methods in feedback theory with applications in high-gain, fast-sampling, and cheap-optimal control, IMA J. Math. Control. Inform. 1 (1984) 147-171. 
[11] D.H. Owens, D. Prätzel-Wolters and A. Ilchmann, Positive-real structure and high-gain adaptive stabilization, IMA J. Math. Control Inform. 4 (1984) 167-181.

[12] D. Prätzel-Wolters, D.H. Owens and A. Ilchmann, Robust stabilization by high gain feedback and switching, Internat. J. Control 49 (1989) 1861-868.
[13] J.C. Willems and C.I. Byrnes, Global adaptive stabilization in the absence of information on the sign of the high frequency gain, in: Lect. Notes in Control and Information Science No. 62 (Springer-Verlag, Berlin, 1984) 49-57. 